\title{
A Robot at Home - How Affect, Technology Commitment, and Personality Traits Influence User Experience in an Intelligent Robotics Apartment
}

\author{
Jasmin Bernotat, Friederike Eyssel, CITEC, Bielefeld University
}

\begin{abstract}
Previous research has shown that user features like affect, personality traits, user gender, technology commitment, perceived ease of technology use, and the feeling of being observed impact human-technology interaction (e.g., [1], [2]). To date, most studies have focused on the influence of user characteristics while interacting with single technical devices such as smart phones, audio players (e.g., [3]), or computers (e.g., [1]). To extend this work, we investigated the influence of individual user characteristics, the perceived ease of task completion, and the feeling of being observed on human-technology interaction and human-robot interaction (HRI) in particular. We explored how participants would solve seven tasks within a smart laboratory apartment. To do so, we collected video data and complemented this analysis with survey data to investigate naïve users' attitudes towards the smart home and the robot. User characteristics such as agreeableness, low negative affect, technology acceptance, low perceived competence regarding technology use, and the perceived ease of task were predictors of positive user experiences within the intelligent robotics apartment. Regression analyses revealed that a positive evaluation of the robot was predicted by positive affect and, to a lesser extent, by technology acceptance. Actual interactions with the robot were predicted by a positive evaluation of the robot and, to a lesser degree, by technology acceptance. Moreover, our findings show that user characteristics and, by tendency, the ease of task impact HRI within an intelligent apartment. Implications for future research on how to investigate the interplay of user and further task characteristics to improve HRI are discussed.
\end{abstract}

\section{INTRODUCTION}

Studying HRI within smart home environments has become an increasingly important research topic in recent years [4], [5], [6]. Psychological aspects such as individual user characteristics as well as context factors like task characteristics determine positive and efficient human-robot interaction: For instance, person variables like user gender [2], affect [7], technology commitment (see [3]), and personality traits [8] clearly improve human-technology interaction. Similarly, the ease of use leads to more positive user experiences (e.g., [1]). Perceived ease of use is based on individual characteristics, such as people's general confidence in performing a task using technology. Nevertheless, it is also related to characteristics of a specific system, like perceptions of external control and objective usability of technology [9]. Moreover, [3] have described the impact of emotions, user attributes, and the given task on user experiences and intentions to use technology.

\footnotetext{
*This research is part of the Cognitive Service Robotics Apartment as Ambient Host (CSRA) project. It has been conducted in the framework of the European Project CODEFROR (FP7 PIRSES-2013-612555) and it was supported by the Cluster of Excellence Cognitive Interaction Technology 'CITEC' (EXC 277) at Bielefeld University, which is funded by the German Research Foundation (DFG).
}

These authors have asked participants to solve tasks with diverse technical devices (e.g., to handle a smart phone, a portable audio player). They found that user attributes (e.g., experience and skills), system properties (e.g., functionality and interface design), and the task characteristics determined the quality of user experience, and therefore, the appraisal of the respective technical device. This, in turn determines the intentions to use the technical devices.

Previous research on user experience has shed light on the impact of user (e.g., [2], [3], [8]), system, and context-related characteristics [2], [3] on human-technology interaction towards single technical systems, but not towards a complex system like an intelligent robotics apartment. For instance, [7] found that high positive affect leads to a positive perception of technology use. The same was found for high endorsement of technology commitment [10], the perceived ease of a given task, and male gender [2]. Likewise, high endorsement of the Big Five personality traits extraversion, conscientiousness [11], [12], agreeableness [11], openness to experience [11, 13], and low endorsement of neuroticism (see [11], [12]) had an effect on human-technology interaction. As a context-related factor, feeling observed had an impact on human-technology interaction (see [14]). Furthermore, only few of these studies have focused on user, system, and context-related characteristics and their influence on HRI within an intelligent apartment. In the smart home context, so far, the technical aspect of integrating various sensors and interfaces have been in the focus rather than examining in depth the psychological aspects of human-system interaction [15].

To fill this research gap, we investigated user characteristics as well as factors related to the system and the context within an intelligent robotics apartment. We expected user characteristics, namely, high positive affect (and low negative affect, respectively) [7], high endorsement of technology commitment (i.e., acceptance, competence, and control) [9], high endorsement of the Big Five factors (BFI-10, [16]) extraversion, conscientiousness [11], [12], agreeableness [15], openness to experience [13], low scores endorsement of neuroticism (see [11], [12]), male gender [2], a high perceived ease of task, and a low feeling of being observed (see [14]) to predict a positive evaluation of the interaction with the intelligent robotics apartment in general (H1) and with the robot in particular (H2). Moreover, we expected the same factors to predict

J. Bernotat is with Center of Excellence Cognitive Interaction Technology, Bielefeld University, Germany (email: jbernotat@cit-ec.uni-bielefeld.de).

F. Eyssel is with Center of Excellence Cognitive Interaction Technology, Bielefeld University, Germany (email: feyssel@cit-ec.uni-bielefeld.de). 
the actual use of the robot during the study (H3). Furthermore, a positive evaluation of the interaction with the intelligent robotics apartment (H4) and the robot in particular (H5) were expected to predict the actual use of the robot during the study.

\section{METHODS}

\section{A. Participants}

63 participants were recruited at a German university campus to take part in this study. 16 individuals had to be excluded from data analysis due to technical problems or because they did not follow the instructions. All participants in the final sample $\left(n=47 ; 26\right.$ females, 21 males; $M_{\text {age }}=25.26$, $S D_{\text {age }}=5.69$; age range: $18-50$ years) were unfamiliar with the intelligent robotics apartment.

\section{B. Procedure}

Firstly, participants gave consent to having their audio and video data recorded. After welcoming them to the intelligent robotics apartment, the experimenter briefly pointed to the robot [17] and the assistant who stayed in the experimental setting for legal reasons and in case the robot needed to be controlled manually (see section robot platform for detailed information about the robot). The intelligent robotics apartment consists of four rooms: a kitchen, a living-room, a private gym, and a bathroom. Subsequently, participants received written information describing seven everyday tasks that had to be solved within the intelligent robotics apartment (see Table 1 for the complete list of tasks). We deliberately selected tasks that are common in everyday life and thus were presumably familiar to our participants and easy to complete. To keep conditions constant, the tasks had to be solved in a given order. Except for Task 7, which had to be solved without using speech, participants were invited to intuitively and freely utilize various approaches to complete the given tasks. For instance, participants were instructed to turn on the light in the hallway of the intelligent robotics apartment (see Table 1, Task 1). They could use speech and gestures to complete the task. However, they were not allowed to operate a light switch to complete the task successfully. Furthermore, participants were told not to use their mobile phones or wrist watches for reference (see Table 1, Tasks 5 and 6). No additional details were given about the available interfaces of the intelligent robotics apartment, functionalities, and how to operate the smart technologies. Using a Wizard-of-Oz approach [18], an experimenter observed participants' behavior from the control room next to the laboratory apartment. When a participant showed 'valid' attempts to solve a given task, the experimenter simulated allegedly autonomous responses of the respective system. Prior to the experiment, we had defined an attempt as 'valid' when participants followed the instructions to show intuitive, but at the same time innovative approaches to solve a given task. That means, when participants showed any goaldirected behavior to complete a task, except using switches (and speech regarding Task 7). For instance, a hand gesture towards the light as a signal to switch it on or off accounted as a valid attempt to complete Task 1 or Task 2 , respectively (Table 1).

After completing all tasks, participants filled in a paperpencil survey that assessed their overall experience during the interaction with the apartment and the robot, respectively. Participants were debriefed in oral and written form and finally received $€ 6$ or research credits for participation.

TABLE I. INTERACTIVE TASKS PERFORMED BY THE PARTICIPANTS.

\begin{tabular}{ll}
\hline No. & \multicolumn{1}{c}{ Tasks } \\
\hline 1 & Turn the hallway light on \\
2 & Turn the hallway light off \\
3 & Listen to music \\
4 & Find out whether a mail has been delivered \\
5 & Find out whether there was a missed phone call \\
6 & Find out the current time \\
7 & Alter the brightness of a floor lamp \\
\hline
\end{tabular}

\section{Materials}

Survey: The positive and negative affect scale [19] encompasses two subscales to measure positive (PA) and negative affect (NA). Participants were provided with 20 adjectives and were asked to rate for each of the emotional states how they felt during their stay in the intelligent robotics apartment. Therefore, a list of ten positive (PA: e.g., "active", "interested", $a=.84$ ) and ten negative adjectives (NA: e.g., "afraid", "nervous", $a=.79$ ) was administered and participants' responses were used to compute mean scores of positive and negative affect, respectively. Technology commitment [20] comprises three subscales that assess the extent to which participants accept new technology (Acceptance: e.g., "I am always interested in using new technology", $a=.82$ ), their perceived competence in using technology (Competence: e.g., "To use technical devices is difficult for me. - I just can't handle it.", $a=.83$ ), and their ability to control technical devices (Control: e.g., "I can control technical devices.", $a=.66$ ). Furthermore, participants completed a 10 -item short version of the Big Five Inventory (BFI-10, [21]) which is based on the (BFI44, [16]). The BFI-10 [21] assesses the five core dimensions of personality: To illustrate, extraversion describes an individual's level of gregariousness and activity. Agreeableness encompasses how trustful and tender-minded a person is. Conscientiousness describes the trait of being reliable and self-disciplined, whereas neurotic people come across as anxious and vulnerable. Openness to experience reflects a person's sense of aesthetics and openness for ideas [16]. We measured all five dimensions using two items per personality factor: extraversion ("I am reserved.", "I am outgoing and sociable.", $a=.79$ ), agreeableness ("I am generally trusting.", "I tend to find faults with others.", $a=.46$ ), conscientiousness ("I tend to be lazy.", "I do a thorough job.", $a=.46$ ), neuroticism ("I am relaxed and handle stress well.", "I get nervous easily."; $a=.59$ ), and openness ("I have few artistic interests.", "I have an active imagination.", $a=.53$ ).

To assess participants' evaluation of their stay in the intelligent robotics apartment, they had to answer two items indicating how pleasant they felt during the interaction with the apartment and while interacting with the robot. Finally, participants had to indicate whether they had difficulties to fulfill the tasks using the technical devices implemented in the intelligent robotics apartment and whether they felt observed by the assistant. All survey items were answered on Likert scales ranging from 1 to 7 (except for PANAS, for which responses 
could range from 1 to 5 and demographics for which open questions were used). High scores indicate a high endorsement of the respective concept.

Robot Platform: The robot used in the current research was a bi-manual robot based on the model "Meka Mobile Manipulator M1" [17]. It is a humanoid system with two dexterous arms and hands, an exchangeable sensor head equipped with camera eyes, and an omnidirectional wheel base. The arms have 7 Degrees of freedom (DOF), the end-effectors are 5 DOF hands, and Meka's torso has 2 DOF [22] (Fig. 1). So, the robot could move its head towards the participants while welcoming them. The robot was programmed to provide assistance in solving the given tasks, e.g., by pointing to objects or by uttering short sentences. To illustrate, when participants asked for the robot's assistance to ask for a mail delivery, it either pointed to the kitchen cupboard or told participants verbally that a mail had been delivered and was placed in the kitchen cupboard (Table 1). The robot was stationed near the kitchen and the living room. It did not navigate within the apartment for safety concerns. An assistant stayed nearby to operate the emergency stop button in case needed.

Video and Audio Data Analysis: To measure and interpret participants' intuitive behavior in the intelligent robotics apartment, audio and video materials were analyzed. This gave us the opportunity to relate participants' behavior to their evaluation of the interaction with the apartment and with the robot. Each of the interactive locations of the apartment were captured, namely kitchen, hallway, and living room. Bathroom and private gym were not captured due to privacy concerns. Thus, task completion did not require to enter these rooms. The first 31 datasets were captured from three different camera perspectives. However, detailed gestures and facial expressions that might reflect user intentions and emotions could not be tracked adequately with this setup. Therefore, a fourth camera was installed for the remaining 32 participants (Fig. 1). System data, such as the Wizard's actions were available with each event being temporally aligned (see [18]). To explain, the Wizard's actions served to identify what was considered as a suitable task solution. The temporal alignment recorded which system was triggered and when it was triggered. Moreover, two raters annotated the video and audio materials manually. They created a coding system to classify participants' approaches during the interaction with the intelligent robotics apartment. These annotations were realized using EUDICO Linguistic Annotator (ELAN 4.9.1, [23], [24]) and enabled statistical data analysis. The coding system contained tiers to assess participants' behavior during the study. One annotation tier documented the course of the study. That means, participants' progress during the study was described (e.g., whether task completion was merely initiated or whether a task was solved successfully) along all the sessions. Participants' methods to approach a task were coded, for example, whether they used speech or gestures to fulfill a given task. For instance, an annotation tier called focus of attention classified which interfaces participants addressed before they completed a task successfully (e.g., first they addressed the robot, but then decided to use a touch screen). Another annotation tier was used to describe which interface participants finally used to complete a given task. Furthermore, some annotation tiers served to document participants' use of speech to control the environment. For instance, whether they asked for advice or whether they addressed an interface to ask for the current time in order to complete the given task (Table 1, Task 6). To validate the coding system and to ensure consistency between raters, eight videos were annotated by both of them. Inter-rater reliabilities indicate high agreements (Cohen's $\kappa$ ranged between .66 and $1.00, p<.001)$. Fig. 1 shows the experimental setup and the Meka robot after a participant completed all tasks successfully (indicated by the floor lamp that is turned on, Task 7, Table 1).

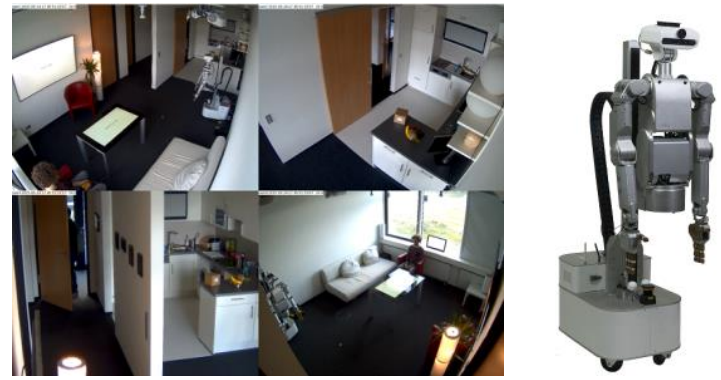

Figure 1. The intelligent robotics apartment from four camera perspectives including the Meka robot (also depicted on the right).

\section{RESULTS}

\section{A. Statistical Analyses}

To test whether user characteristics (i.e., participants' scores on PA, NA, the BFI factors, technology commitment subscales acceptance, competence, control, gender), the perceived ease of task which is related to user and system features, and as a context factor, the subjective feeling of being observed by the assistant would influence the evaluation of the interaction with the apartment and the robot, standard multiple regression analyses were performed. In the first regression analysis, we examined these characteristics and the context factor as predictors for the evaluation of the interaction with the apartment (H1). In the second regression analysis, the same predictors were used to indicate a positive evaluation of the interaction with the robot $(\mathrm{H} 2)$. To investigate which factors lead to the use of the robot $(\mathrm{H} 3)$, a binary logistic regression analysis was run with the use of the robot during the study as a dichotomous variable predicted by PA, NA, BFI factors, technology commitment subscales acceptance, competence, control, gender, perceived ease of task, the feeling of being observed by the assistant, and participants' evaluations of the interaction with the apartment (H4) and with the robot (H5). Whether participants used the robot during the study was analyzed according to the video annotations. The actual use of the robot was dummy-coded with 0 representing participants who did not actually use the robot and 1 representing participants who used the robot during the study. In all analyses, gender was dummy-coded with 0 representing male participants and 1 representing female participants.

$T$-tests against the neutral scale midpoint were performed to identify whether participants' endorsements of the respective constructs were different from the neutral scale midpoint. This allowed an interpretation whether participants' endorsement of a respective construct was relative high or low. 


\section{B. Descriptive Statistics}

Preliminary Analyses: Table 2 shows that participants felt rather good during their stay in the apartment, as indicated by PA and NA. They scored significantly higher on positive $\left(M_{\mathrm{PA}}=3.26, S D_{\mathrm{PA}}=0.64\right)$ than on negative affect $\left(M_{\mathrm{NA}}=1.50\right.$, $\left.S D_{\mathrm{NA}}=0.42\right), t(46)=13.66, p<.001, d=3.25$ Analyzing participants' attitudes towards technology, we found high average scores on the three subscales of technology commitment. The exploration of participants' BFI personality traits showed that participants' scores on openness to experience, agreeableness, and conscientiousness were significantly above scale midpoint. Means on extraversion and neuroticism did not differ from scale midpoint. Participants perceived the given tasks as easy to fulfill and felt marginally observed by the assistant during the study. On average, participants perceived the interaction with the apartment positively. However, the contact with the robot was rated as rather neutral.

TABLE II. MEANS AND Standard DeViations of the MEASURes TESTED AGAINST SCALE MIDPOINTS.

\begin{tabular}{lcccc}
\hline \multicolumn{1}{c}{ Construct } & $\boldsymbol{M}$ & $\boldsymbol{S D}$ & $\boldsymbol{t}$ & $\boldsymbol{p}$ \\
\hline PA & 3.26 & 0.64 & 2.82 & .007 \\
NA & 1.50 & 0.42 & -24.74 & .001 \\
Acceptance & 5.04 & 1.29 & 5.51 & $<.001$ \\
Competence & 5.80 & 1.15 & 10.71 & $<.001$ \\
Control & 5.34 & 0.97 & 9.41 & $<.001$ \\
Extraversion & 4.48 & 1.65 & 1.99 & .052 \\
Agreeableness & 4.63 & 1.31 & 3.29 & .002 \\
Openness & 5.06 & 1.28 & 5.70 & $<.001$ \\
Conscientiousness & 4.47 & 1.19 & 2.71 & .010 \\
Neuroticism & 4.05 & 1.40 & 0.26 & .796 \\
Ease of task & 5.26 & 1.34 & 6.41 & $<.001$ \\
Feeling observed & 4.49 & 1.98 & 1.70 & .096 \\
Evaluation apartment & 5.64 & 1.55 & 7.24 & $<.001$ \\
Evaluation robot & 4.48 & 1.75 & 1.86 & .070 \\
\hline
\end{tabular}

Note. $M=$ mean, $S D=$ standard deviation, $t=t$-value, degrees of freedom $(\mathrm{df})=46, p=$ probability, scale midpoints: PANAS $=3$, all other constructs $=4$.

Frequency of participants who used the robot: Analyses of the video annotations revealed that a total of 16 ( 5 male users, 11 female users) participants interacted with the robot during the study. On average, these participants contacted it about four times during the study $\left(M_{\text {robot-interaction }}=3.69\right.$, $S D_{\text {robot-interaction }}=1.76$ ).

\section{Users' Evaluation of the Apartment}

To test whether user characteristics and system and context related factors would influence participants' evaluation of the interaction with the apartment (H1), a standard multiple regression analysis with $\mathrm{PA}$, NA, technology commitment subscales, BFI factors, and participants' gender, ease of task, and feeling observed as predictors was conducted. The prediction model was statistically significant, $F(13,33)=9.60$, $p<.001$, and accounted for about $79 \%$ of the variance of the evaluation of the interaction with the apartment, $R^{2}=.79$, Adjusted $R^{2}=.71, f=1.95$. A positive evaluation of the interaction with the apartment was predicted by lower levels of NA and higher levels of acceptance, competence, agreeableness, and by tendency ease of task. All other predictors were not statistically significant (Table 3 ).
TABLE III. RESULTS OF A STANDARD MULTIPLE REGRESSION.

\begin{tabular}{lcccc}
\hline \multicolumn{1}{c}{ Model } & $\boldsymbol{B}$ & SE $\boldsymbol{B}$ & $\boldsymbol{\beta}$ & $\boldsymbol{r}$ \\
\hline Constant & $4.43 \dagger$ & 2.39 & & \\
PA & 0.41 & 0.25 & 0.17 & $.54 * * *$ \\
NA & $-1.33^{*}$ & 0.50 & -0.36 & $-.55^{* * * *}$ \\
Acceptance & $0.55^{* * *}$ & 0.14 & 0.45 & $.43^{*}$ \\
Competence & $-0.56^{* *}$ & 0.16 & -0.41 & -.07 \\
Control & 0.07 & 0.16 & 0.04 & .13 \\
Extraversion & -0.08 & 0.10 & -0.81 & .16 \\
Agreeableness & $0.27^{*}$ & 0.11 & 0.22 & .15 \\
Conscientiousness & -0.21 & 0.14 & -0.16 & -.04 \\
Neuroticism & 0.05 & 0.11 & 0.05 & -.06 \\
Openness & 0.10 & 0.14 & 0.08 & -.05 \\
Gender & 0.32 & 0.39 & 0.10 & .01 \\
Ease of task & $0.33^{\dagger}$ & 0.17 & 0.28 & $.65 * * *$ \\
Feeling observed & -0.13 & 0.08 & -0.17 & $-.44 *$ \\
\hline
\end{tabular}

Note. $B=$ unstandardized beta, SE $B=$ standard error in beta, $\beta=$ standardized coefficient,

$r=$ Pearson correlation with the dependent variable. ${ }^{\dagger} p<.10 .{ }^{*} p<.05 .{ }^{* * *} p<.001$.

\section{User's Evaluation of the Robot}

To test whether user and task characteristics would predict a positive user experience while interacting with the robot (H2), we performed a standard multiple regression analysis in the same manner as in section $\mathrm{C}$, but with the evaluation of the interaction with the robot as a dependent variable. The prediction model was statistically significant, $F(13,32)=2.30$, $p=.027$, and accounted for about $48 \%$ of the variance of the evaluation of the interaction with the robot, $R^{2}=.48$, Adjusted $R^{2}=.27, f=.97$. Interestingly, the prediction model revealed that PA was the only statistically significant predictor, while technology commitment subscale acceptance was a marginally significant predictor. All other predictors were not statistically significant (Table 4).

TABLE IV. RESULTS OF A STANDARD MULTIPLE REGRESSION.

\begin{tabular}{lcccc}
\hline \multicolumn{1}{c}{ Model } & $\boldsymbol{B}$ & SE $\boldsymbol{B}$ & $\boldsymbol{\beta}$ & $\boldsymbol{r}$ \\
\hline Constant & -2.71 & 4.27 & & \\
PA & $1.25^{*}$ & 0.47 & 0.46 & $.51^{* * *}$ \\
NA & -1.33 & 0.88 & -0.32 & $-.38^{*}$ \\
Acceptance & $0.44^{\dagger}$ & 0.24 & 0.32 & .17 \\
Competence & -0.12 & 0.28 & -0.08 & -.06 \\
Control & 0.25 & 0.29 & 0.14 & $.22^{\dagger}$ \\
Extraversion & 0.15 & 0.18 & 0.14 & $.25^{*}$ \\
Agreeableness & 0.11 & 0.19 & 0.08 & .07 \\
Conscientiousness & 0.19 & 0.25 & 0.13 & .14 \\
Neuroticism & 0.11 & 0.20 & 0.09 & -.01 \\
Openness & 0.04 & 0.26 & 0.03 & .13 \\
Gender & 1.10 & 0.69 & 0.32 & .19 \\
Ease of task & -0.24 & 0.31 & -0.18 & $.38^{*}$ \\
Feeling observed & 0.04 & 0.15 & 0.05 & $-.27^{*}$ \\
\hline
\end{tabular}

Note. $B=$ unstandardized beta, SE $B=$ standard error in beta, $\beta=$ standardized coefficient, $r=$ Pearson correlation with the dependent variable. ${ }^{\dagger} p<.10 .{ }^{*} p<.05 .{ }^{* * *} p<.001$.

\section{E. Actual interactions with the robot}

Some participants directly addressed the robot during their stay in the apartment. The actual use of the robot during the apartment stay was used as a dichotomous dependent variable with PA, NA, technology commitment subscales, BFI-factors, gender, the perceived ease of tasks, the feeling of being observed (H3), the evaluation of the interaction with the apartment (H4) and with the robot (H5) as predictors. Hence, a binary logistic regression analysis was run. The overall model was not statistically significant, $\chi^{2}(15)=20.92, p=.139$, 
Nagelkerke's $R^{2}=.50$. However, tests of model effects displayed that the evaluation of the robot was a statistically significant predictor of actual use of the robot, Wald's $\chi^{2}(1)=4.56, p=.033$. Moreover, technology commitment subscale acceptance was a marginally significant predictor, Wald's $\chi^{2}(1)=3.43, p=.064$ (Table 5).

TABLE V. RESULTS OF A BINARY LOGISTIC REGRESSION.

\begin{tabular}{|c|c|c|c|c|c|}
\hline \multirow{2}{*}{ Model } & \multirow{2}{*}{$\boldsymbol{B}$} & \multirow{2}{*}{ SE $B$} & \multirow{2}{*}{$\begin{array}{c}\operatorname{Exp} \\
(B)\end{array}$} & \multicolumn{2}{|c|}{ CI for $\operatorname{Exp}(B)$} \\
\hline & & & & $L L$ & $\boldsymbol{U L}$ \\
\hline Constant & -8.03 & 11.18 & 0.00 & & \\
\hline Gender & -2.15 & 1.56 & 0.12 & 0.01 & 2.50 \\
\hline $\mathrm{PA}$ & 1.21 & 1.12 & 3.36 & 0.38 & 30.01 \\
\hline NA & 0.66 & 2.16 & 1.94 & 0.03 & 133.52 \\
\hline Acceptance $^{\dagger}$ & -1.40 & 0.76 & 0.25 & 0.06 & 1.09 \\
\hline Competence & -0.10 & 0.74 & 0.90 & 0.21 & 3.88 \\
\hline Control & 0.45 & 0.57 & 1.57 & 0.52 & 4.75 \\
\hline Extraversion & 0.22 & 0.36 & 1.24 & 0.62 & 2.50 \\
\hline Agreeableness & 0.18 & 0.43 & 1.20 & 0.51 & 2.80 \\
\hline Conscientiousness & -0.19 & 0.63 & 0.83 & 0.24 & 2.85 \\
\hline Neuroticism & 0.03 & 0.45 & 1.03 & 0.42 & 2.48 \\
\hline Openness & 0.13 & 0.68 & 1.14 & 0.30 & 4.36 \\
\hline Ease of task & 0.39 & 0.62 & 1.48 & 0.44 & 4.93 \\
\hline Feeling observed & 0.46 & 0.36 & 1.58 & 0.79 & 3.18 \\
\hline Evaluation apartment & -0.28 & 0.64 & 0.76 & 0.22 & 2.67 \\
\hline Evaluation robot* & 0.92 & 0.43 & 2.52 & 1.08 & 5.88 \\
\hline
\end{tabular}

Note. $\mathrm{B}=$ regression coefficient, $\mathrm{SE} \mathrm{B}=$ standard error in beta, $\operatorname{Exp}(\mathrm{B})=$ odds ratio, $\mathrm{CI}=$ confidence interval, $\mathrm{LL}=$ lower limit, $\mathrm{UL}=$ upper limit. $\dagger \mathrm{p}<.10 .{ }^{*} \mathrm{p}<.05$.

\section{DISCUSSION}

In the current study, we investigated whether user characteristics and system and context related factors influence participants' experience with an intelligent robotics apartment in general and with a robot in particular. Furthermore, we explored whether these factors would positively predict the use of the robot during the study. Participants had to solve seven mundane tasks in the apartment (Table 1). They were free to choose any interface they considered suitable to complete those tasks. That is, participants could use the robot or any other available device in the apartment. The interaction with the intelligent environment was followed by a questionnaire. In this questionnaire, we assessed user characteristics, namely, positive and negative affect, BFI factors, technology commitment as well as the perceived ease of a task which is related to user and system characteristics and, as a context factor, whether participants felt observed by the assistant during the study. Furthermore, participants had to indicate how comfortable they felt during the interaction with the apartment and with the robot. Our results show that participants were rather high in positive affect and low in negative affect, respectively. On average, participants reported high technology commitment on all three subscales technology acceptance, competence in its use, and control over technology. Participants scored relatively high on openness to experience, agreeableness, and conscientiousness. However, the technology commitment subscale control and the Big Five factors showed only moderate to low Cronbach's alphas which indicate the reliabilities of the scales. Despite this, we kept using the technology commitment subscales instead of analyzing technology commitment in general because they provide more detailed information [25]. Regarding the Big Five personality traits, future studies should consider the BFI-44 [16] full list of items. Furthermore, participants experienced the interaction with the apartment positively and the contact with the robot was evaluated rather neutrally. Participants had no difficulty to solve the given tasks and felt only slightly observed by the assistant during the study.

One of our main concerns was to investigate user, system, and context-related factors as predictors for a positive experience with the apartment. The results partly confirmed our hypotheses. Low negative affect, high technology acceptance and low competence in technology use, high agreeableness, and marginally the ease of task were predictors for a positive evaluation of the interaction with the apartment. However, PA, self-reported control over technology, the BFI factors openness to experience, extraversion, conscientiousness, and neuroticism as well as the impression of being observed by the assistant during the study did not predict a positive evaluation of the interaction with the intelligent robotics apartment. Similarly, some previous studies found no effects of openness to experience on the perceived usefulness and the intention to use a technical system [11], [12]. Hence, this might emphasize the need for usefulness of technology. Due to the lack of an introduction to the intelligent robotics apartment, people might not have seen any benefit of interacting with the apartment and the robot. The finding that low NA, but not PA predicted a positive evaluation of the interaction with the apartment is surprising. Previous research pointed to the role of positive affect and positive emotions in the use of technology [7]. However, it was not pointed to the absence of negative affect. The influence of the technology commitment subscales acceptance and competence on a positive experience with the apartment is similar to [7] who state that computer-self-efficacy, which is related to competence in technology use, leads to higher positive affect, more outcome expectations, and consequently to the use of a computer. Accordingly, the ease of task was a marginally significant predictor of a positive experience with the apartment (see also [2]). Thus, the perceived ease of task completion might have been the reason why those who rated themselves low on competence of technology evaluated the apartment positively. The finding, that agreeableness influenced participants' evaluation of the interaction with the apartment might be explained by [16]. The authors found that people high on agreeableness are trusting. Hence, the relation between agreeableness and trust might have played a role within the intelligent robotics apartment. [26] has pointed out that trust leads to a decrease in perceived risk, an increase in perceived usefulness of technical systems, enhanced ease of use, and higher intentions to use a technical system. Thus, future studies should consider the role of trust on user experience in an intelligent robotics apartment further.

Moreover, we investigated whether user, system, and context-related factors would indicate a positive evaluation of the interaction with the robot and predict the use of the robot within the intelligent robotics apartment. Only partly in line with our hypotheses, positive affect and marginally, the technology commitment subscale acceptance, predicted a positive 
experience with the robot during the study. Further, participants who evaluated the robot positively and, interestingly those who showed a lesser technology acceptance, tended to use the robot during the study. Noteworthy, only a minority of participants interacted with the robot to solve a given task. However, those who had contacted the robot once kept using it. Others stated they did not use the robot because they did not know what it was capable to do. This might again have caused a lack of perceived usefulness of (and trust in) the robot's assistance. This could explain why some participants failed to fulfill the tasks. Maybe they did not consider the robot as a suitable interface. Nevertheless, the results of the current study emphasize the importance of negative and positive affect and technology acceptance, especially towards robots during human-robot interaction. Our results imply that people ideally need an introduction to technical systems to allow an appropriate estimation of whether a task can be solved easily, effectively, and without risks by using the respective system. This is again in line with [26]. The author points to the role of trust towards technical devices to enhance users' acceptance and intention to use technological systems.

In conclusion, our results show that user characteristics and system and context-related factors influence people's behavior and their evaluation of an intelligent robotics apartment and a robot. Importantly, these results demonstrate which factors lead to the actual use of a robot. These findings also emphasize the importance of understanding intelligent environments as systems of technical devices. At the same time, researchers and developers should be aware that user experience and behavior are determined by different factors depending on the device they are exposed to.

\section{REFERENCES}

[1] Venkatesh, V. and Davis, F. D., "A theoretical extension of the technology acceptance model: Four longitudinal field studies," Manage. Sci., vol. 46, Feb. 2000, pp. 186-204.

[2] V. Venkatesh and M. G. Morris, "Why don't men ever stop to ask for directions? Gender, social influence, and their role in technology acceptance and usage behavior," MIS Quarterly, vol. 24, Mar. 2000, pp. 115-139.

[3] M. Thüring and S. Mahlke, "Usability, aesthetics and emotions in human-technology interaction," Int. J. Psychol., vol. 42, Aug. 2007, pp. 253-264.

[4] J. Pineau, M. Montemerlo, M. Pollack, N. Roy, and S. Thrun, "Towards robotic assistants in nursing homes: Challenges and results," Robot. Auton. Syst., vol 42, Mar. 2003, pp. 271-281.

[5] M. Chan, E. Campo, D. Estève, and J.-Y. Fourniols, "Smart homes -Current features and future perspectives," Maturitas, vol. 64, Oct. 2009, pp. 90-97.

[6] E. Kaasinen, T. Kymäläinen, M. Niemelä, T. Olsson, M. Kanerva, and V. Ikonen, "A user-centric view of intelligent environments: user expectations, user experience and user role in building intelligent environments," Computers, vol. 2, Dec. 2012, pp. 1-33.

[7] D. R. Compeau and C. A. Higgins, "Computer self-efficacy: development of a measure and initial test," MIS Quart., vol. 19, Jun. 1995, pp. 189-211.

[8] T. Correa, A.W. Hinsley, and H.G. de Zúñiga, "Who interacts on the web?: The intersection of users' personality and social media use," Comput. Hum. Behav., vol. 26, Mar. 2010, pp. 247-253.

[9] V. Venkatesh, and H. Bala, "Technology acceptance model 3 and a research agenda on interventions," Decision Sci., vol. 39, May 2008, pp. 273-315.
[10] F. D. Davis, "Perceived usefulness, perceived ease of use, and user acceptance of information technology," MIS Quarterly, vol. 13, Sept 1989, pp. 319-340.

[11] S. Devaraj, R. F. Easley, and J. M. Crant, "Research note- how does personality matter? Relating the five-factor model to technology acceptance and use," Inform Syst Res, vol. 19, Mar. 2008, pp. 93-105.

[12] T. Barnett, A. W. Pearson, and F. W. Kellermans, "Five-factor model personality traits as predictors," Eur. J. Inform. Syst., vol. 24, Jul. 2015, pp. 374-390, DOI: 10.1057/ejis.2014.10

[13] J. C. McElroy, A. R. Hendrickson, A. M. Townsend, and S. M. DeMarie, "Dispositional factors in internet use: Personality versus cognitive style", MIS Quarterly, vol. 31, Dec. 2007, pp. 809-820.

[14] C. Bartneck, T. Bleeker, J. Bun, P. Fens, L. and Riet, L., "The influence of robot anthropomorphism on the feelings of embarrassment when interacting with robots, " Paladyn, J. Behav Robotics, vol. 1, Jan. 2010, pp. 109-115.

[15] P. Holthaus, C. Leichsenring, J. Bernotat, V. Richter, M. Pohling, B., ... and S. Wrede, „How to address smart homes with a social robot? A multi-modal corpus of user interactions with an intelligent environment," Proceedings of the 10th Language Resources and Evaluation Conference, May 2016, pp. 3440-3446.

[16] P. T. Costa and R. R. McCrae, R. R., "Normal personality assessment in clinical practice: The NEO Personality Inventory", Psychological assessment, vol. 4, Mar. 1992, pp. 5-13.

[17] Meka robotics announces mobile manipulator with kinect and ROS IEEE Spectrum: http://spectrum.ieee.org/automaton/ robotics/humanoids/meka-robotics-announces-mobile-manipulatorwith-kinect-and-ros. Accessed: 2016-10-03.

[18] J. F. Kelley, "An iterative design methodology for user-friendly natural language office information applications," ACM Transactions on Information Systems, vol. 2, Jan. 1984, pp. 26-41.

[19] H. W. Krohne, B. Egloff, C. W. Kohlmann, and A. Tausch, A., „Untersuchungen mit einer deutschen Version der 'Positive and Negative Affect Schedule' (PANAS) “ [Investigation of a German version of the positive and negative affect schedule (PANAS)], Diagnostica, vol. 42, Apr. 1996, pp. 139-156.

[20] F. J. Neyer, J. Felber, and C. Gebhardt, „Entwicklung und Validierung einer Kurzskala zur Erfassung von Technikbereitschaft," Entwicklung und Validierung einer Kurzskala zur Erfassung von Technikbereitschaft [Development and evaluation of a short-scale to assess technology commitment], Diagnostica, vol. 58, Apr. 2012, pp. 87-99.

[21] B. Rammstedt and O. P. John, "Measuring personality in one minute or less: A 10-item short version of the Big Five Inventory in English and German," J. Res. Pers., vol. 41, Feb. 2007, pp. 203-212.

[22] MeKa Robotics: A. Edsinger and J. Weger, San Francisco, 2006.

[23] ELAN - Max Planck Institute for Psycholinguistics, The Language Archive, Nijmegen, The Netherlands: http://tla.mpi.nl/tools/tlatools/elan/.

[24] H. Sloetjes and P. Wittenburg, "Annotation by category: ELAN and ISO DCR", Proceedings of the 6th Language Resources and Evaluation Conference, Jul. 2008, pp. 816-820.

[25] N. Schmitt, "Uses and abuses of coefficient alpha," Psychological Assessment, vol. 8, Dec. 1996, pp. 350-353.

[26] P. A. Pavlou, "Consumer acceptance of electronic commerce: integrating trust and risk with the technology acceptance model," Int. J. Electron. Comm., vol. 7, Apr. 2003, pp. 101-134. 